\title{
Stellar Coronal and Wind Models: Impact on Exoplanets
}

\author{
Aline A. Vidotto
}

\begin{abstract}
Surface magnetism is believed to be the main driver of coronal heating and stellar wind acceleration. Coronae are believed to be formed by plasma confined in closed magnetic coronal loops of the stars, with winds mainly originating in open magnetic field line regions. In this Chapter, we review some basic properties of stellar coronae and winds and present some existing models. In the last part of this Chapter, we discuss the effects of coronal winds on exoplanets.
\end{abstract}

\section{Introduction}

About $90 \%$ of the currently known exoplanets orbit around low-mass stars. These stars $\left(0.1 \lesssim M_{\star} / M_{\odot} \lesssim 1.3\right)$, while in the main-sequence phase, have convective interiors that vary in extension as a function of the stellar mass. Below $\sim 0.4 M_{\odot}$, these stars are fully convective. Above this mass threshold, there is an appearance of a radiative core, whose size is larger for more massive stars. In turn, the convective part of the star is limited to the outer layers and becomes progressively smaller as one goes towards more massive stars. At $\sim 1.3 M_{\odot}$, the outer convective envelope is already very small.

As convection is one of the key ingredients in the generation of magnetic fields, main-sequence low-mass stars have. This magnetism gives rise to a multitude of phenomena, from small and localised features (spots, active regions, prominences) to large-scale ones (global magnetism, coronal holes, helmet streamers).

Surface magnetism is also believed to be the main driver of coronal heating and stellar wind acceleration. However, at present, there is no consensus of the basic physical mechanisms involved in these processes. Even for the Sun, heating of the solar corona and acceleration of the solar wind are still currently being debated, with possible scenarios relating to propagation and dissipation of waves and turbulence

School of Physics, Trinity College Dublin, the University of Dublin, Dublin-2, Ireland, e-mail: aline.vidotto@tcd.ie 
in open magnetic flux tubes and/or reconnection between open and closed magnetic flux tubes (Cranmer 2009).

In this Chapter, we start by reviewing basic properties of stellar coronae and winds. We then present a review of some existing models. The last part of this Chapter is dedicated to the impact of coronal winds on exoplanets.

\section{Observationally-derived properties of stellar coronae}

Low-mass stars harbour hot coronae with average temperatures on the order of $10^{6}$ $-10^{7} \mathrm{~K}$ (Guedel 2004, Telleschi et al. 2005, Johnstone and Guedel 2015). The hot stellar coronae are detected in X-ray wavelengths (e.g. Pizzolato et al. 2003; Guedel 2004; Telleschi et al. 2005; Maggio et al. 2011; Wright et al. 2011; Scandariato et al. 2013: Pillitteri et al. 2014; Johnstone and Guedel 2015), during both quiescent and flaring states. Coronae are believed to be formed by plasma confined in closed magnetic coronal loops of the stars. An indication that coronae have indeed their origins in stellar magnetism comes from the observed correlation between X-ray emission and stellar magnetic fields (Pevtsov et al. 2003, Vidotto et al. 2014a). In this Section, we highlight a few observed properties of stellar coronae. An interested reader will find comprehensive reviews of X-ray in, e.g., Guedel (2004); Guedel and Nazé (2009); Testa et al. (2015).

$\mathrm{X}$-ray coronae and stellar rotation: Earlier studies have shown the connection between and chromospheric activity (Kraft 1967). Similarly, X-ray emission has also been recognised to correlate with stellar rotation, with the exception of fastrotating stars (e.g. Pallavicini et al. 1981; Pizzolato et al.|2003; Jeffries et al.|2011; Wright et al. 2011; Reiners et al. 2014). For this reason, the activity-rotation relation is usually divided into two parts. Fast-rotating stars have $\mathrm{X}$-ray emission that is roughly independent of rotation. They are in the so called saturated regime. These stars have X-ray luminosities that account for about $0.1 \%$ of their bolometric luminosities. For slower rotators, in the unsaturated regime, $\mathrm{X}$-ray luminosities increases with rotation rate $\Omega_{\star}$ as (Reiners et al.2014)

$$
L_{x} \propto \Omega_{\star}^{2.01 \pm 0.05} .
$$

The rotation rate at which stars transition from unsaturated to saturated regimes corresponds to (Johnstone and Guedel 2015)

$$
\frac{\Omega_{\star, \text { sat }}}{\Omega_{\odot}} \simeq 13.53\left(\frac{M_{\star}}{M_{\odot}}\right)^{1.08}
$$

where $\Omega_{\odot}=2.67 \times 10^{-6} \mathrm{rad} \mathrm{s}^{-1}$. The saturation threshold is mass-dependent, with lower-mass stars transitioning from the saturated to the unsaturated regime at lower rotation rates. As rotation decreases with the square-root of the age of stars (Sku- 
manich 1972), stars in the lowest-mass range (e.g., M dwarfs) remain saturated even at relatively old ages (note also that these stars have longer lifetimes).

Another observed link between rotation and X-ray emission is seen in X-ray lightcurves. Because X-ray emission arises in closed magnetic coronal loops and since the distribution of closed/open magnetic field line regions at the surface of the star is inhomogeneous, stars can also show rotational modulations in X-ray (Hussain et al. 2005, 2007).

$\mathrm{X}$-ray coronae and temperatures It has also been shown that stars with hot coronae have high X-ray emission (e.g. Telleschi et al. 2005). For low-mass mainsequence stars, there is a tight relation between X-ray flux $F_{x}$ and average $\tilde{T}_{c}$ (Johnstone and Guedel 2015)

$$
F_{x}=0.9\left(\frac{\tilde{T}_{c}}{10^{6} \mathrm{~K}}\right)^{3.8} \operatorname{erg~cm}^{-2} \mathrm{~s}^{-1}
$$

This empirical relation is useful for estimating the average coronal temperature of stars, once $F_{x}$ is known. $F_{x}$ can either be determined observationally or by using the rotation-activity relation (e.g., Equation 11. As we will see in this Chapter, the temperature is an unknown in the models. Models that relate the temperature of the wind to the temperature of the corona can benefit from the empirical relation (3).

$\mathrm{X}$-ray coronae and magnetism The link between coronae and magnetism has long been identified. For this reason, X-ray emission is often used as a proxy for stellar magnetism. One way to validate this is by confronting observed values of X-ray luminosities/fluxes with observations of stellar magnetism.

Two methods are mostly used to measure stellar magnetism. The Zeemaninduced line broadening of unpolarised light (Stokes I), or Zeeman broadening (ZB) technique (e.g., Solanki 1994; Saar 1996; Johns-Krull et al.| 1999; Johns-Krull 2007; Saar 2001: Reiners et al. 2009), yields estimates of the average of the total unsigned surface field strength $\left\langle\left|B_{I}\right|\right\rangle$ (small- and large-scale structures). This technique does not provide information of the topology of the field. The () technique (Stokes V), on the other hand, is able to reconstruct the intensity and topology of the stellar magnetic field (e.g., Donati and Brown 1997; Donati and Landstreet 2009; Morin et al. 2013), but cannot reconstruct the small-scale field component, which is missed within the resolution element of the reconstructed ZDI maps (Johnstone et al.|2010; Arzoumanian et al. 2011, Lang et al. 2014). As a consequence, the ZDI magnetic maps are limited to measuring large-scale magnetic field.

Pevtsov et al. (2003) found that the $\mathrm{X}$-ray luminosities are related to the unsigned magnetic fluxes $\Phi_{I}$ measured by the $\mathrm{ZB}$ technique

$$
L_{x} \propto \Phi_{I}^{1.13 \pm 0.05}
$$

where

$$
\Phi_{I}=\left\langle\left|B_{I}\right|\right\rangle 4 \pi R_{\star}^{2} .
$$


To derive this relation, Pevtsov et al. (2003) considered magnetic field observations of the Sun (X-ray bright points, active regions, quiet Sun and integrated solar disk) and pre- and main-sequence stars. This empirical relation can be seen in Figure 1 1 , spanning about 12 orders of magnitude in magnetic flux.

Similarly, Vidotto et al. (2014a) found that

$$
L_{x} \propto \Phi_{V}^{0.913 \pm 0.054}
$$

where

$$
\Phi_{V}=\left\langle\left|B_{V}\right|\right\rangle 4 \pi R_{\star}^{2}
$$

is the unsigned magnetic flux as derived from the technique (i.e., only contains the large-scale component of the stellar magnetic field). To be consistent with the method from Pevtsov et al. (2003), the relation above considers both main-sequence stars and pre-main sequence (accreting) stars. The slope found by Vidotto et al. (2014a) is consistent to the nearly linear trend found by Pevtsov et al. (2003).

Figure 1 月1 1 shows that the pre-main sequence stars (open circles) are underluminous as compared to the empirical fit (solid line). When considering only the sample of $16 \mathrm{G}, \mathrm{K}$ and $\mathrm{M}$ dwarf stars (i.e., no solar data nor accreting PMS stars), Pevtsov et al. (2003) found that $L_{x}^{\text {(dwarfs) }} \propto \Phi_{I}^{0.98 \pm 0.19}$. Considering the same types of objects, the relation derived from ZDI data yields $L_{X}^{\text {(dwarfs) }} \propto \Phi_{V}^{1.80 \pm 0.20}$ (based on a larger sample of 61 dwarf stars). This is shown in Figure 1 1 p. Given the larger errors in the exponents of the fits, both relations are consistent to each other within $3 \sigma$. Still, this is a topic worth of future investigation. For example, finding a different power law for $\Phi_{V}$ and $\Phi_{I}$ might clarify on how the small-scale and large-scale field structures contribute to $\mathrm{X}$-ray emission.

\section{Observationally-derived properties of stellar winds}

Low-mass stars undergo mass loss through winds during their entire lives. Contrary to the Sun, in which the solar wind can be probed in situ, the existence of winds around low-mass stars is known indirectly, e.g., from the observed rotational evolution of stars (e.g., Bouvier et al. 2014). Measuring the wind $\dot{M}$ of cool, low-mass stars is challenging, as these winds are rarefied and difficult to be directly detected.

Attempts to measure low mass star's winds have been done through radio observations of their free-free thermal emission at radio wavelengths (e.g., van den Oord and Doyle 1997, Gaidos et al. 2000, Villadsen et al. 2014) and through X-ray observations of the emission generated when ionised wind particles exchange charges

\footnotetext{
${ }^{1}$ The data provided in Figure $1 \mathrm{a}$ are from: Donati et al. 1999 2003 $2008 \mathrm{a}$ c b $2010 \mathrm{a}$ b $2011 \mathrm{a}$ b c 2012 2013); Marsden et al. (2006 2011); Catala et al. (2007); Morin et al. (2008a b 2010); Petit et al. (2008 2009); Hussain et al. (2009); Fares et al. (2009| 2010||2012||2013); Morgenthaler et al. (2011 2012); Waite et al. (2011 2015 2017); do Nascimento et al. (2016); Folsom et al. (2016) and from Petit et al. in prep.
} 

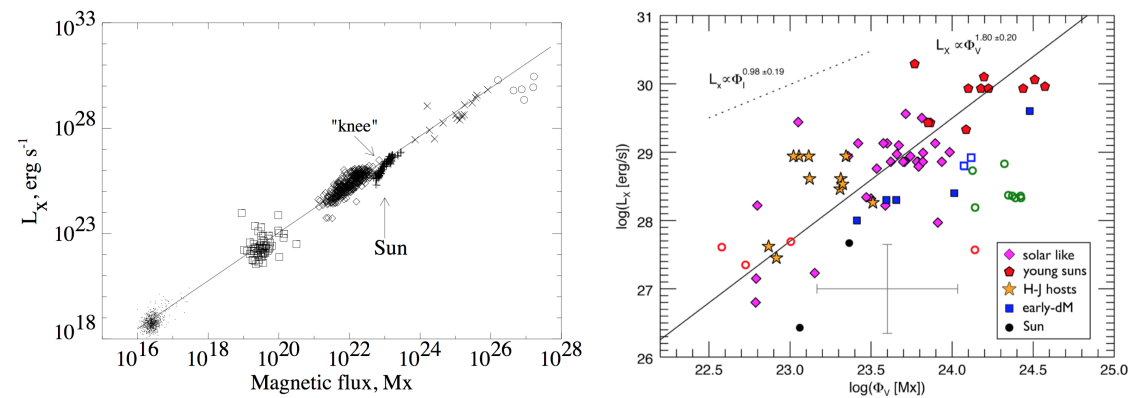

Fig. 1 (a) Relation between the X-ray luminosities and the unsigned magnetic fluxes $\Phi_{I}$ measured by the Zeeman broadening technique (Pevtsov et al.2003). Reproduced by permission of the AAS. (b) The same as in (a) but for magnetic field measurements done with the Zeeman Doppler imaging technique. The different symbols mainly indicate different types of surveys. The solid line shows the empirical fit through the data, while the dashed line (at an arbitrary vertical offset) is indicative of the slope found by the Zeeman broadening technique, when considering the $16 \mathrm{G}, \mathrm{K}, \mathrm{M}$ stars. From Vidotto et al. (2014 MNRAS, 441, 2361).

with neutral atoms of the interstellar medium (Wargelin and Drake 2002). Other attempts involve the observations of coronal radio flares (Lim and White 1996) or the accretion of wind material from a cool low-mass star to a white dwarf in binary systems (Debes 2006, Parsons et al. 2012). So far, the indirect method proposed by Wood et al. (2001), which involves reconstruction of stellar Lyman- $\alpha$ line (see below), has been the most successful one, enabling estimates of $\dot{M}$ for about a dozen dwarf stars. To illustrate the challenging aspects of measuring, we show in Table 1 tentative measurements of $\dot{M}$ of the closest star to us, namely Proxima Centauri. Recently, the interest in understanding Proxima Centauri has increased due to the discovery of a terrestrial type planet orbiting in its habitable zone (Anglada-Escudé et al. 2016).

Table 1 Characteristics of Proxima Centauri and its wind.

\begin{tabular}{lll}
\hline Physical property & Value & Reference \\
\hline mass $\left(M_{\odot}\right)$ & 0.123 & $\mathrm{a}$ \\
radius $\left(R_{\odot}\right)$ & 0.141 & $\mathrm{~b}, \mathrm{c}$ \\
rotation period (days) & $\sim 83$ & $\mathrm{c}$ \\
$F_{x}\left(10^{6} \mathrm{erg} \mathrm{cm}^{-2} \mathrm{~s}^{-1}\right)$ & $\sim 1.2$ & $\mathrm{~d}$ \\
$\tilde{T}_{c}\left(10^{6} \mathrm{~K}\right)$ & 2.7 & $\mathrm{e}$ \\
spectral type & $\mathrm{M} 5.5$ & $\mathrm{f}$ \\
total magnetic flux $(\mathrm{G})$ & 600 & $\mathrm{~g}$ \\
$\dot{M}\left(\dot{M}_{\odot}=2 \times 10^{-14} \mathrm{M}_{\odot} \mathrm{yr}^{-1}\right)$ & $<350$ & $\mathrm{~h}$ \\
$\ldots$ & $<14$ & $\mathrm{i}$ \\
$\ldots$ & 0.2 & $\mathrm{f}$ \\
\hline
\end{tabular}

a: Ribas et al. (2016); b: Demory et al. (2009); c: Anglada-Escudé et al. (2016); d: Wood (2004); e: Güdel et al. (2004); f: Wood et al.(2001); g: Reiners and Basri (2008); h: Lim et al. (1996); i: Wargelin and Drake(2002). 
Wood et al.'s method explains the excess absorption observed in the blue wing of the Lyman- $\alpha$ line as caused by the hydrogen wall that forms during the interaction between the stellar wind and the interstellar medium. Wood et al. (2002, 2005) found a relation between the mass-loss rate per unit surface area $A_{\star}=4 \pi R_{\star}^{2}$ and the for low-mass stars

$$
\dot{M} / 4 \pi R_{\star}^{2} \propto F_{x}^{1.34} .
$$

As X-ray emission is related to rotation and rotation can be related to stellar ages, Equation $(6)$ implies that younger stars have higher $\dot{M}$ than older ones. Also, Equation (6) is only valid for $F_{x} \lesssim 10^{6} \mathrm{erg} \mathrm{cm}^{-2} \mathrm{~s}^{-1}$ or ages $\gtrsim 600 \mathrm{Myr}$. Figure 2 compiles the mass-loss rates derived by Wood (2004); Wood et al. (2014).

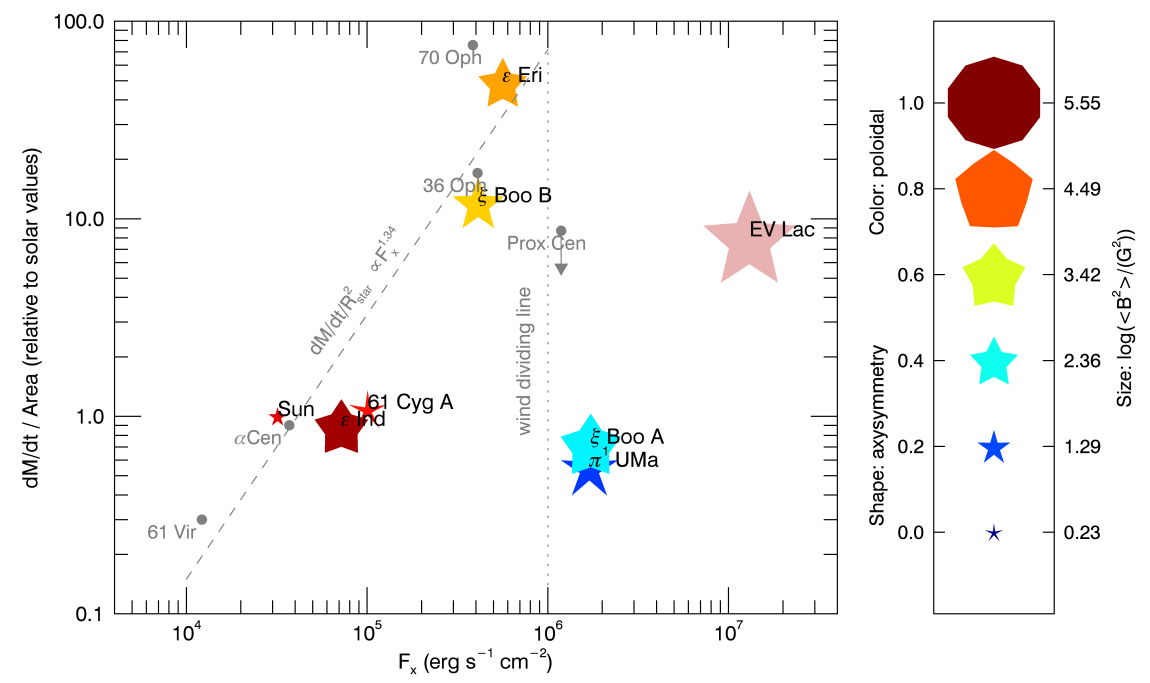

Fig. 2 Compilation of the mass-loss rates derived by Wood (2004); Wood et al. (2014) as a function of. Grey circles are stars that do not yet have measurements of their surface magnetic fields with ZDI. For the other systems, symbol sizes are proportional to the magnetic energy $\log \left\langle B^{2}\right\rangle$, their colours indicate the fractional poloidal energy (ranging from deep red for purely poloidal field $f_{\mathrm{pol}}=1$ to blue for purely toroidal field $f_{\mathrm{pol}}=0$ ), and their shapes indicate the fraction of axisymmetry of the poloidal component (ranging from a decagon for purely axisymmetric field $f_{\text {axi }}=1$ to a point-shaped star for $f_{\text {axi }}=0$ ). The $\dot{M}-F_{x}$ relation (Eq. 6 is shown as a dashed line and the WDL at $F_{x}=10^{6} \mathrm{erg} \mathrm{cm} \mathrm{cm}^{-2} \mathrm{~s}^{-1}$ is shown as a dotted line. The maps used to produce this figure are from Morin et al. (2008b); Morgenthaler et al. (2012); Jeffers et al. (2014); Boro Saikia et al. (2016); Vidotto (2016); Petit et al in prep; and Boisse et al in prep. Figure based on Vidotto et al. (2016, MNRAS, 455L, 52).

The break in the $\dot{M}-F_{x}$ relation found by Wood et al. (2005) for active stars with $F_{x} \gtrsim 10^{6} \mathrm{erg} \mathrm{cm}^{-2} \mathrm{~s}^{-1}$ has been suggested to be caused by the topology of that would inhibit the wind generation (Wood et al. 2005; Wood and Linsky|2010). Vidotto et al. (2016) analysed this hypothesis with a sub-sample of stars observed by Wood et al that also had observationally-derived large-scale magnetic fields with ZDI. These authors did not find any particular evidence that the magnetic field characteristics 
show an abrupt change at the wind dividing line (WDL, at $F_{x} \sim 10^{6} \mathrm{erg} \mathrm{cm}^{-2} \mathrm{~s}^{-1}$ ). In general, solar-type stars to the right of the WDL (namely $\xi$ Boo A and $\pi^{1} \mathrm{UMa}$ ) have higher fractional toroidal fields (blueish points in Fig.2), but no break or sharp transition was found.

Very active stars, in particular, show variability in their properties on timescales on the order of a few years and can, for example, jump between states with highly toroidal fields and mostly poloidal fields (Petit et al. 2009: Morgenthaler et al.|2012, Jeffers et al. 2014, Boro Saikia et al. 2015). If magnetic fields are to affect stellar winds, significant scatter in the points to the right of the WDL are to be expected.

\section{Models of stellar coronal winds}

Studies of the solar wind have provided insights into the winds of low-mass stars. However, as there is still no consensus of the basic physical mechanisms involved in the acceleration of the solar wind (Cranmer 2009), this uncertainty also propagates to models of stellar winds. The are believed to be magnetically-driven, in which coupling between stellar magnetism and convection transports free magnetic energy, which in turn is converted into thermal energy in the upper atmosphere of stars (Matsumoto and Suzuki 2014), giving rise to a hot corona (illustrated in Figure 3 for the solar atmosphere). The scale height of X-ray emitting stellar corona is likely to vary with the properties of the star (Jardine 2004: Guedel 2004). A possible scenario to convert magnetic into thermal energy involves the dissipation of waves and turbulence (e.g., Holzer et al. 1983, Cranmer 2008; Cranmer and Saar 2011; Suzuki et al. 2013; Matsumoto and Suzuki 2014). In addition to depositing energy, waves also transfer momentum to the wind, accelerating it (e.g., Vidotto and Jatenco-Pereira 2006). In general, two modelling approaches are used in the study of the hot coronal winds of low-mass stars. We describe them next.

Self-consistent heating/acceleration mechanism The first approach involves a more rigorous computation of the wave energy and momentum transfer, i.e., the computations are done from "first principles" (e.g., Hollweg 1973, Holzer et al. 1983; Hartmann and MacGregor 1980; Jatenco-Pereira and Opher 1989, Vidotto and Jatenco-Pereira 2006, Falceta-Gonçalves et al. 2006, Cranmer 2008, Cranmer and Saar 2011; Suzuki et al. 2013). In these models, the increase in temperature from the colder photosphere to the hotter corona arises naturally in the solution of the equations as does the wind acceleration. Most of the models that treat the acceleration starting from the photosphere have been limited to analytical, one- and twodimensional solutions, as, depending on the level of details of the physics involved in the wind acceleration/heating mechanism, models can become computationally intensive. In particular, a challenging numerical aspect is the large density contrast between the photosphere and the rarefied corona (e.g. Matsumoto and Suzuki 2012). Additionally, models are usually restricted to the inner-most part of the wind and they usually adopt simple topologies for the stellar magnetic field. 


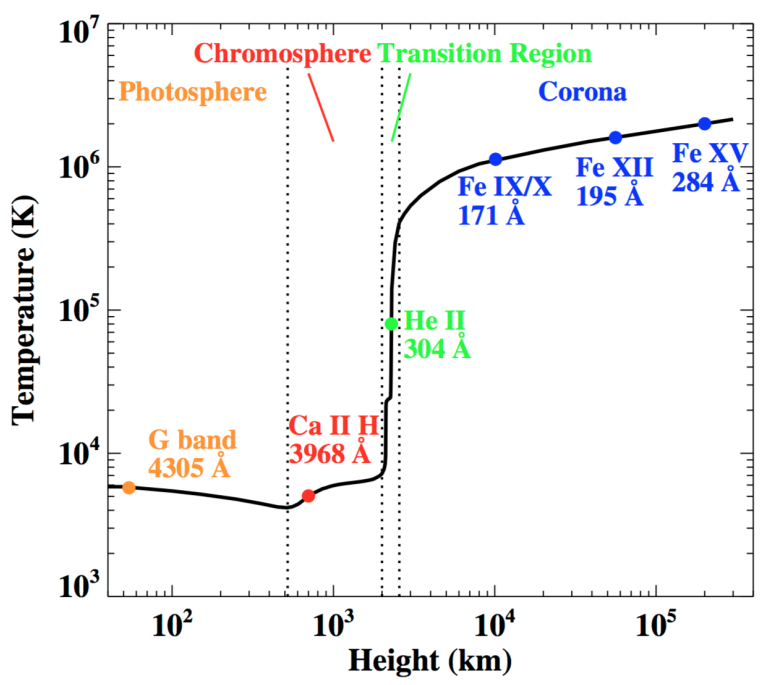

Fig. 3 Temperature variation with height for the solar atmosphere. Credit: Yang et al., A\&A, 501, 745,2009 , reproduced with permission (C)ESO.

Global wind models The second approach adopts a simplified energy equation, usually assuming the wind is described by a polytropic equation of state. In the latter, the thermal pressure $p_{\text {th }}$ is related to density $n$ as $p_{\text {th }} \propto n^{\gamma}$, where $\gamma$ is known as the . In these models, the computation often starts at the point where the temperature has already reached coronal values $\sim 10^{6} \mathrm{~K}$ (e.g., Mestel 1968 ; Pneuman and Kopp 1971: Tsinganos and Low 1989; Washimi and Shibata 1993; Keppens and Goedbloed 2000; Lima et al. 2001; Matt and Pudritz 2005; Matt et al.|2012;:Vidotto et al. 2009b|a, 2010b; Cohen et al. 2009, 2010; Pinto et al. 2011; Johnstone et al. 2015a b, Réville et al. 2015, Lüftinger et al. 2015). This approach ignores the physical reason of what led temperatures to increase from photospheric to coronal values. On the other hand, equations are simpler, allowing us to perform three-dimensional numerical simulations of "global" stellar winds, i.e., extending out to large distances from the star (Vidotto et al. 2009b a, 2010b, 2012, 2014b, 2015; Cohen et al. 2009, 2010; Jardine et al. 2013; Llama et al.|2013, Strugarek et al. 2015; Nicholson et al. 2016).

More recently, there have been efforts in developing a hybrid approach that combines the two approaches described above to study . In these hybrid models, a phenomenological approach of the (solar-based) wave heating mechanism is implemented in three-dimensional simulations of solar/stellar winds, starting from the upper chromosphere (van der Holst et al. 2010, 2014; Sokolov et al. 2013; Garraffo et al. 2015; Alvarado-Gómez et al. 2016). These models present a step forward in the modelling of winds of low-mass stars, as they, for example, do not need to impose a polytropic index to mimic energy deposition in the wind. However, there are still some parameters that need to be imposed a priori, such as the energy flux of 
waves at the inner boundary and its dissipation length scale, as discussed in Sokolov et al. (2013). In the case of the solar wind, these free parameters can be constrained from observations (Sokolov et al.|2013; van der Holst et al.|2014).

One of the advantages of global wind models is that the numerical grid can extend out to large distances, allowing us to characterise the conditions around exoplanets (Vidotto et al. 2009a, 2010b, 2012, 2015; Cohen et al. 2011a b; Llama et al. 2013, Nicholson et al. 2016; Vidotto and Donati 2017). The characterisation of the stellar wind (i.e., the ) is important to quantify the wind (magnetic and particles) effects on exoplanets, as we will discuss later. The global wind models can also incorporate more complex magnetic field topologies, including those derived from observations of (ZDI maps). The magnetic maps are imposed as boundary conditions at the stellar wind base. The magnetic field lines are then extrapolated into the computational domain (e.g., corona, astrosphere), initially assuming the field is in its lowest energy state (i.e., a potential field). With the interaction of the stellar wind particles, the magnetic field becomes stressed. The self-consistent interaction between magnetic field lines and stellar wind particles are let to evolve, until a relaxed solution is found (for more details, see e.g., Vidotto et al.2014b). The left panel of Figure47illustrates the solution of the stellar wind model of the planet-hosting star HD 189733, computed using the observationally-derived ZDI magnetic map from Fares et al. (2010). Colour-coded is the total wind pressure (thermal, magnetic and ram pressures) relative to the solar wind pressure at the Earth's orbit. The right panel of Figure 4 shows, in the background, the X-ray emission of the hot, quiescent corona of the star due to thermal free-free radiation (Llama et al. 2013). Coronal comes mainly from flaring magnetic loops with different sizes. As the small-scale magnetic structure is not resolved in ZDI observations, the X-ray emission computed in Llama et al. (2013) captures only the quiescent corona and, as such, provide a lower limit for the emission. Overlaid to the X-ray image in Figure 4 is the velocity of the stellar wind at the position of the orbit of HD 189733b (indicated in the left panel by the black circumference).

\section{Stellar wind effects on exoplanets}

The majority of exoplanets known nowadays orbit stars at considerably close distances. These close orbits are not represented in our solar system. The giant exoplanets at close-in orbits are also known as hot-Jupiters. The system presented in Figure 4 and studied by Llama et al.(2013), for instance, hosts a hot-Jupiter, namely HD 189733b. The solid black line shown in the left panel of Figure 4 4 represents its orbital radius, at about $\sim 8.7 R_{\star}$. From the values of the stellar wind pressure at the orbital position of this exoplanet, we see that the environment surrounding hotJupiters have considerably different physical conditions than those around the solar system planets. densities, magnetic field intensities, temperatures, and pressures all decay with distance, albeit with different dependencies (see also Vidotto et al. 2015). This means that the interactions between winds or stellar magnetic field lines 

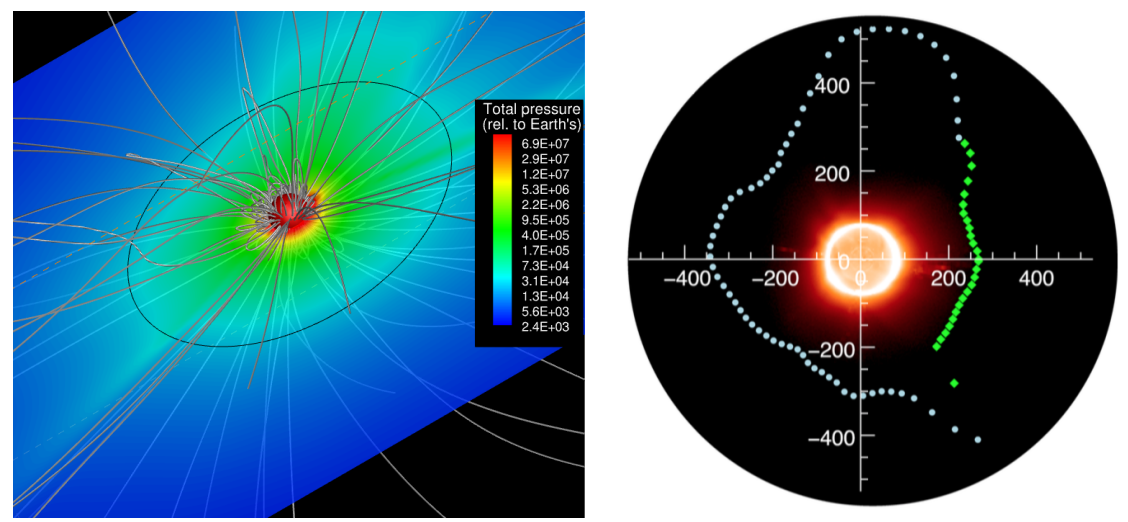

Fig. 4 Left: Stellar wind model of the planet-hosting star HD189733, computed using the observationally-derived ZDI magnetic map from Fares et al. (2010). The total wind pressure is shown in colour, in a slice through the equatorial plane, and is given in units relative to the solar wind pressure at the Earth's orbit. The orbital radius of HD 189733b is represented by the black circumference. Right: The X-ray emission of the quiescent corona (i.e., it neglects the non-thermal emission of flares and associated to small-scale magnetic field components) is shown in the background. In the foreground, it is shown the velocity of the stellar wind at the position of the orbit of HD 189733b. Image from Llama et al. (2013, MNRAS, 436, 2179).

are strongest for close-in planets. Despite the fact that stellar winds are still accelerating and therefore have usually low velocities at the position of close-in exoplanets, the orbital velocities of these planets are large due to the $1 / \sqrt{r}$-dependence of Keplerian velocities. can, therefore, have supersonic orbital velocities in the azimuthal direction (Vidotto et al. 2010a). This means that the relative velocity of the close-in planet through the wind of its host star can be as large as that of the solar wind impacting on the Earth's magnetosphere. The main difference between these two scenarios is the direction of the velocity vector, which has a large azimuthal (radial) component in the former (latter) case.

Because of the large stellar wind densities at the position of , the relative motion of the planet through the wind of the star results in large ram pressures. This, for example, can give rise to the formation of bow shocks surrounding exoplanets (Vidotto et al. 2010a, 2011a; Llama et al. 2013; Bisikalo et al. 2013). In a recent numerical study, Matsakos et al. (2015) showed that other structures, such as cometary-type tails, and inspiraling accretion streams, can also appear as a result of the interactions between stellar winds and planetary magnetic fields/outflows (see also Lai et al. 2010; Li et al. 2010; Bourrier and Lecavelier des Etangs 2013; Bourrier et al. 2016).

One consequence of the large pressure of the stellar wind environment around exoplanets is that it can constrain the sizes of planetary magnetospheres. The standoff distance between the planetary surface and the magnetopause is set by pressure balance. At the planet-stellar wind interaction zone, pressure balance between the stellar wind (left-hand side) and planetary magnetosphere (right-hand side) can be 
written as

$$
\rho \Delta u^{2}+p_{\text {th }}+\frac{B_{r_{\text {orb }}}^{2}}{8 \pi} \simeq \frac{B_{p, r_{M}}^{2}}{8 \pi},
$$

where $\rho, p_{\text {th }}$ and $B_{r_{\text {orb }}}$ are the density, thermal pressure and magnetic field strength of the stellar wind at the position of the planetary orbit, $\Delta u$ is the relative velocity of the planet through the wind of the host-star and $B_{p, r_{M}}$ is the planetary magnetic field intensity at a distance $r_{M}$ from the planet centre. Eq. (7) neglects the planetary thermal pressure component on the right side. Because of the exponential decay of planetary densities, at the height of a few planetary radii, thermal pressure is usually negligible compared to the planetary magnetic pressure. We further take the to be dipolar, such that $B_{p, r_{M}}=B_{p \text {,eq }}\left(R_{p} / r_{M}\right)^{3}$, where $R_{p}$ is the planetary radius and $B_{p, \text { eq }}$ its surface magnetic field at the equator (i.e., half the value of the intensity at the magnetic pole). For a planetary dipolar axis aligned with the rotation axis of the star, the magnetospheric size of the planet is given by

$$
\frac{r_{M}}{R_{p}}=\left[\frac{B_{p, \mathrm{eq}}^{2}}{8 \pi\left(\rho \Delta u^{2}+p_{\mathrm{th}}\right)+B_{r_{\mathrm{orb}}}^{2}}\right]^{1 / 6} .
$$

Therefore, a large stellar wind pressure (ram, thermal and/or magnetic, shown in the denominator of Equation 8 ) acts to reduce the size of for a given planetary magnetisation (see also, e.g., Ip et al. 2004, Zieger et al. 2006; Lovelace et al. 2008, Lanza 2009, Vidotto et al. 2009a, 2010b, 2012, 2011b; Sterenborg et al. 2011; Khodachenko et al. 2012, Buzasi 2013). This can have important effects on the habitability of exoplanets, including terrestrial type planets orbiting inside the habitable zones of their host stars (Grießmeier et al. 2005, 2009; Khodachenko et al. 2007; Lammer et al. 2007, Vidotto et al. 2011b c, Zendejas et al. 2010; Vidotto et al. 2013; See et al. 2014; Ribas et al. 2016). The magnetosphere acts to deflect the stellar wind particles, preventing its direct interaction with planetary atmospheres and, therefore, atmospheric erosion. If the is too small, part of the atmosphere of the planet becomes exposed to the interaction with stellar wind particles. Lammer et al. (2007) suggested that, for a magnetosphere to protect the atmosphere of planets, planets are required to have magnetospheric sizes of $\gtrsim 2 R_{p}$.

\section{Effects on potentially habitable planets around M dwarf stars}

Due to a combination of a that is closer to the star and the technologies currently adopted in exoplanet searches, have been the prime targets for detecting terrestrial planets in the potentially life-bearing region around the star. dM stars, however, can have significantly high magnetic fields, in particular, when they are young, fast rotating and, thus, active (Donati et al. 2008a; Morin et al. 2008b, 2010, Reiners et al. 2009). For example, the late dM star WX UMa, have large-scale magnetic fields of up to $4 \mathrm{kG}$ (Morin et al. 2010), significantly larger than the large-scale 
solar magnetism of several G (Vidotto 2016). With time, the magnetic field intensity of dM stars decay (Vidotto et al. 2014a). However, dM stars are known to remain active over long timescales (on the order of Gyr,West et al.|2008). This implies that the environment surrounding exoplanets that orbit $\mathrm{dM}$ stars should remain highly magnetised for long periods of time.

Vidotto et al. (2013) studied the effects of the high magnetisation of dM stars in setting the sizes of the magnetospheres of (hypothetical) Earth-like planets orbiting in their habitable zones. Using a sample of $15 \mathrm{dM}$ stars with measured surface magnetic fields and assuming planets to have a similar terrestrial magnetisation, they showed that these hypothetical Earths would have magnetospheres that extend as low as $1 R_{p}\left(1.7 R_{p}\right)$ and at most up to $6 R_{p}\left(11.7 R_{p}\right)$, for planets orbiting in the inner (outer) edge of the habitable zone. The magnetospheric size of Proxima $\mathrm{b}$ (orbiting around a M5.5V star, see Table 1 h has been estimated to extend up to about 1.3 to $7 R_{p}$ (Ribas et al. 2016). For comparison, the Earth's magnetosphere is located at $r_{M} \simeq 10-15 R_{\oplus}$ (Bagenal 1992), showing that Earth-like planets with similar terrestrial magnetisation orbiting active dM stars present smaller than that of the Earth. If exoplanets lack a protective magnetic shield, this potentially implies that these planets can lose a significant fraction of their atmospheres (Zuluaga et al. 2013).

\section{Effects on potentially habitable planets orbiting young stars}

Sun-like stars are observed to emit larger X-ray and extreme ultra-violet (EUV) fluxes at a younger age (e.g. Guinan et al.|2003; Ribas et al.|2005, 2010). The large stellar irradiation can heat the outer atmospheres of exoplanets that become more extended and more susceptible to evaporation (e.g. Lammer et al. 2003, Baraffe et al. 2004: Sanz-Forcada et al. 2011). Planets can receive an enhanced flux of energetic photons if they are orbiting at close distances to their stars and/or if they are orbiting around young and more active stars.

In addition to larger X-ray and EUV fluxes, young and more active stars also harbour more intense magnetic fields and winds with larger $\dot{M}$. Relation (6), between $\dot{M}$ and $F_{x}$, has important consequences, for example, for the evolution of the young solar System. Extrapolations using Eq. (6) suggest that the $700 \mathrm{Myr}-\mathrm{Sun}$ would have had $\dot{M}$ that is $\sim 100$ times larger than the current solar mass-loss rate $\dot{M}_{\odot}=2 \times 10^{14} \mathrm{M}_{\odot} \mathrm{yr}^{-1}$ (Wood et al. 2005). This could explain the loss of the Martian atmosphere as due to erosion caused by the stronger wind of the young Sun (Wood 2004).

Through modelling of the wind of the solar twin $\kappa$ Ceti, do Nascimento et al. (2016) computed the magnetospheric size of the . $\kappa$ Ceti is believed to be a good representation of our Sun at an age of about $400-600 \mathrm{Myr}$, roughly the time that life started emerging on our planet. To calculate the magnetospheric size of the (hypothetical) Earth-twin, we make use of Equation (8). For a similar present-day magnetisation of the Earth, do Nascimento et al. (2016) estimated the size of the 
magnetosphere of the to be about $5 R_{p}$. Contrary to Mars, which does not host a significant magnetic field and therefore no appreciable magnetosphere, the relatively large size of the early Earth's magnetosphere may have been the reason that prevented the volatile losses from Earth and created conditions to support life.

\section{Final remarks}

In this Chapter, we presented one aspect of how stars can affect their surrounding planets. We concentrated on the effects that stellar winds might have on exoplanets. We only presented case studies in which the exoplanet hosts a protective magnetic field, which prevents a direct interaction between the stellar wind and the planetary outer atmosphere. Non-magnetised exoplanets are believed to undergo significant atmospheric erosion in short timescales. However, exoplanetary magnetic fields have not yet been directly observed and so far have only been elusively probed (e.g. Shkolnik et al. 2008; Vidotto et al. 2010a, Kislyakova et al. 2014). Due to the diversity of stellar and exoplanetary properties, of the architectures of exoplanetary systems and the complex nature of stellar wind-planet interaction, progress in this field is likely to come through different angles. This is another example of how exoplanetary studies are becoming more multi-disciplinary, where important physical insights can only be gained from efforts arising from different areas of Astrophysics, such as stellar, solar, and planetary Physics, and astrobiology.

\section{References}

Alvarado-Gómez JD, Hussain GAJ, Cohen O et al. (2016) Simulating the environment around planet-hosting stars. I. Coronal structure. A\&A588:A28

Anglada-Escudé G, Amado PJ, Barnes J et al. (2016) A terrestrial planet candidate in a temperate orbit around Proxima Centauri. Nature536:437-440

Arzoumanian D, Jardine M, Donati J, Morin J Johnstone C (2011) The contribution of star-spots to coronal structure. MNRAS410:2472-2480

Bagenal F (1992) Giant planet magnetospheres. Annual Review of Earth and Planetary Sciences 20:289-328

Baraffe I, Selsis F, Chabrier G et al. (2004) The effect of evaporation on the evolution of close-in giant planets. A\&A419:L13-L16

Bisikalo D, Kaygorodov P, Ionov D et al. (2013) Three-dimensional Gas Dynamic Simulation of the Interaction between the Exoplanet WASP-12b and its Host Star. ApJ764:19

Boro Saikia S, Jeffers SV, Petit P et al. (2015) Variable magnetic field geometry of the young sun HN Pegasi (HD 206860). A\&A573:A17

Boro Saikia S, Jeffers SV, Morin J et al. (2016) A solar-like magnetic cycle on the mature K-dwarf 61 Cygni A (HD 201091). A\&A594:A29

Bourrier V Lecavelier des Etangs A (2013) 3D model of hydrogen atmospheric escape from HD 209458b and HD 189733b: radiative blow-out and stellar wind interactions. A\&A557:A124 
Bourrier V, Lecavelier des Etangs A, Ehrenreich D, Tanaka YA Vidotto AA (2016) An evaporating planet in the wind: stellar wind interactions with the radiatively braked exosphere of GJ $436 \mathrm{~b}$. A\&A591:A121

Bouvier J, Matt SP, Mohanty S et al. (2014) Angular Momentum Evolution of Young Low-Mass Stars and Brown Dwarfs: Observations and Theory. Protostars and Planets VI pp 433-450

Buzasi D (2013) Stellar Magnetic Fields as a Heating Source for Extrasolar Giant Planets. ApJ765:L25

Catala C, Donati JF, Shkolnik E, Bohlender D Alecian E (2007) The magnetic field of the planethosting star $\tau$ Bootis. MNRAS374:L42-L46

Cohen O, Drake JJ, Kashyap VL Gombosi TI (2009) On the Effect of Magnetic Spots on Stellar Winds and Angular Momentum Loss. ApJ699:1501

Cohen O, Drake JJ, Kashyap VL, Hussain GAJ Gombosi TI (2010) The Coronal Structure of AB Doradus. ApJ721:80-89

Cohen O, Kashyap VL, Drake JJ et al. (2011a) The Dynamics of Stellar Coronae Harboring Hot Jupiters. I. A Time-dependent Magnetohydrodynamic Simulation of the Interplanetary Environment in the HD 189733 Planetary System. ApJ733:67-+

Cohen O, Kashyap VL, Drake JJ, Sokolov IV Gombosi TI (2011b) The Dynamics of Stellar Coronae Harboring Hot Jupiters. II. A Space Weather Event on a Hot Jupiter. ApJ738:166-+

Cranmer SR (2008) Turbulence-driven Polar Winds from T Tauri Stars Energized by Magnetospheric Accretion. ApJ689:316-334

Cranmer SR (2009) Coronal Holes. Living Reviews in Solar Physics 6:3-+

Cranmer SR Saar SH (2011) Testing a Predictive Theoretical Model for the Mass Loss Rates of Cool Stars. ApJ741:54

Debes JH (2006) Measuring M Dwarf Winds with DAZ White Dwarfs. ApJ652:636-642

Demory BO, Ségransan D, Forveille T et al. (2009) Mass-radius relation of low and very low-mass stars revisited with the VLTI. A\&A505:205-215

do Nascimento JD Jr, Vidotto AA, Petit P et al. (2016) Magnetic Field and Wind of Kappa Ceti: Toward the Planetary Habitability of the Young Sun When Life Arose on Earth. ApJ820:L15

Donati J Landstreet JD (2009) Magnetic Fields of Nondegenerate Stars. ARA\&A47:333-370

Donati J, Morin J, Petit P et al. (2008a) Large-scale magnetic topologies of early M dwarfs. MNRAS390:545-560

Donati J, Skelly MB, Bouvier J et al. (2010a) Magnetospheric accretion and spin-down of the prototypical classical T Tauri star AA Tau. MNRAS409:1347-1361

Donati J, Skelly MB, Bouvier J et al. (2010b) Complex magnetic topology and strong differential rotation on the low-mass T Tauri star V2247 Oph. MNRAS402:1426-1436

Donati JF Brown SF (1997) Zeeman-Doppler imaging of active stars. V. Sensitivity of maximum entropy magnetic maps to field orientation. A\&A326:1135-1142

Donati JF, Collier Cameron A, Hussain GAJ Semel M (1999) Magnetic topology and prominence patterns on AB Doradus. MNRAS302:437-456

Donati JF, Collier Cameron A, Semel M et al. (2003) Dynamo processes and activity cycles of the active stars AB Doradus, LQ Hydrae and HR 1099. MNRAS345:1145-1186

Donati JF, Jardine MM, Gregory SG et al. (2008b) Magnetospheric accretion on the T Tauri star BP Tauri. MNRAS386:1234-1251

Donati JF, Moutou C, Farès R et al. (2008c) Magnetic cycles of the planet-hosting star $\tau$ Bootis. MNRAS385:1179-1185

Donati JF, Bouvier J, Walter FM et al. (2011a) Non-stationary dynamo and magnetospheric accretion processes of the classical T Tauri star V2129 Oph. MNRAS412:2454-2468

Donati JF, Gregory SG, Alencar SHP et al. (2011b) The large-scale magnetic field and poleward mass accretion of the classical T Tauri star TW Hya. MNRAS417:472-487

Donati JF, Gregory SG, Montmerle T et al. (2011c) The close classical T Tauri binary V4046 Sgr: complex magnetic fields and distributed mass accretion. MNRAS417:1747-1759

Donati JF, Gregory SG, Alencar SHP et al. (2012) Magnetometry of the classical T Tauri star GQ Lup: non-stationary dynamos and spin evolution of young Suns. MNRAS425:2948-2963 
Donati JF, Gregory SG, Alencar SHP et al. (2013) Magnetospheric accretion on the fully convective classical T Tauri star DN Tau. MNRAS436:881-897

Falceta-Gonçalves D, Vidotto AA Jatenco-Pereira V (2006) On the magnetic structure and wind parameter profiles of Alfvén wave driven winds in late-type supergiant stars. MNRAS368:1145-1150

Fares R, Donati J, Moutou C et al. (2009) Magnetic cycles of the planet-hosting star $\tau$ Bootis - II. A second magnetic polarity reversal. MNRAS398:1383-1391

Fares R, Donati J, Moutou C et al. (2010) Searching for star-planet interactions within the magnetosphere of HD189733. MNRAS406:409-419

Fares R, Donati JF, Moutou C et al. (2012) Magnetic field, differential rotation and activity of the hot-Jupiter-hosting star HD 179949. MNRAS423:1006-1017

Fares R, Moutou C, Donati JF et al. (2013) A small survey of the magnetic fields of planet-host stars. MNRAS435:1451-1462

Folsom CP, Petit P, Bouvier J et al. (2016) The evolution of surface magnetic fields in young solartype stars - I. The first 250 Myr. MNRAS457:580-607

Gaidos EJ, Guedel M Blake GA (2000) The faint young Sun paradox: An observational test of an alternative solar model. Geophys Res Lett27:501-504

Garraffo C, Drake JJ Cohen O (2015) The Dependence of Stellar Mass and Angular Momentum Losses on Latitude and the Interaction of Active Region and Dipolar Magnetic Fields. ApJ 813:40

Grießmeier JM, Stadelmann A, Motschmann U et al. (2005) Cosmic Ray Impact on Extrasolar Earth-Like Planets in Close-in Habitable Zones. Astrobiology 5:587-603

Grießmeier JM, Stadelmann A, Grenfell JL, Lammer H Motschmann U (2009) On the protection of extrasolar Earth-like planets around K/M stars against galactic cosmic rays. Icarus 199:526535

Güdel M, Audard M, Reale F, Skinner SL Linsky JL (2004) Flares from small to large: X-ray spectroscopy of Proxima Centauri with XMM-Newton. A\&A416:713-732

Guedel M (2004) X-ray astronomy of stellar coronae. A\&A Rev12:71-237

Guedel M Nazé Y (2009) X-ray spectroscopy of stars. A\&A Rev17:309-408

Guinan EF, Ribas I Harper GM (2003) Far-Ultraviolet Emissions of the Sun in Time: Probing Solar Magnetic Activity and Effects on Evolution of Paleoplanetary Atmospheres. ApJ594:561-572

Hartmann L MacGregor KB (1980) Momentum and energy deposition in late-type stellar atmospheres and winds. ApJ242:260-282

Hollweg JV (1973) ALFVtN Waves in a Two-Fluid Model of the Solar Wind. ApJ181:547-566

Holzer TE, Fla T Leer E (1983) Alfven waves in stellar winds. ApJ 275:808-835

Hussain GAJ, Brickhouse NS, Dupree AK et al. (2005) Inferring Coronal Structure from X-Ray Light Curves and Doppler Shifts: A Chandra Study of AB Doradus. ApJ621:999-1008

Hussain GAJ, Jardine M, Donati JF et al. (2007) The coronal structure of AB Doradus determined from contemporaneous Doppler imaging and X-ray spectroscopy. MNRAS377:1488-1502

Hussain GAJ, Collier Cameron A, Jardine MM et al. (2009) Surface magnetic fields on two accreting TTauri stars: CVCha and CRCha. MNRAS398:189-200

Ip WH, Kopp A Hu JH (2004) On the Star-Magnetosphere Interaction of Close-in Exoplanets. ApJ602:L53-L56

Jardine M (2004) Coronal stripping in supersaturated stars. A\&A414:L5-L8

Jardine M, Vidotto AA, van Ballegooijen A et al. (2013) Influence of surface stressing on stellar coronae and winds. MNRAS431:528-538

Jatenco-Pereira V Opher R (1989) Effect of diverging magnetic fields on mass loss in late-type giant stars. A\&A209:327-336

Jeffers SV, Petit P, Marsden SC et al. (2014) $\varepsilon$ Eridani: an active K dwarf and a planet hosting star?. The variability of its large-scale magnetic field topology. A\&A569:A79

Jeffries RD, Jackson RJ, Briggs KR, Evans PA Pye JP (2011) Investigating coronal saturation and supersaturation in fast-rotating M-dwarf stars. MNRAS411:2099-2112

Johns-Krull CM (2007) The Magnetic Fields of Classical T Tauri Stars. ApJ664:975-985 
Johns-Krull CM, Valenti JA, Hatzes AP Kanaan A (1999) Spectropolarimetry of Magnetospheric Accretion on the Classical T Tauri Star BP Tauri. ApJ510:L41-L44

Johnstone C, Jardine M Mackay DH (2010) Modelling stellar coronae from surface magnetograms: the role of missing magnetic flux. MNRAS404:101-109

Johnstone CP Guedel M (2015) The coronal temperatures of low-mass main-sequence stars. A\&A578:A129

Johnstone CP, Guedel M, Brott I Lüftinger T (2015a) Stellar winds on the main-sequence. II. The evolution of rotation and winds. A\&A577:A28

Johnstone CP, Guedel M, Lüftinger T, Toth G Brott I (2015b) Stellar winds on the main-sequence. I. Wind model. A\&A577:A27

Keppens R Goedbloed JP (2000) Stellar Winds, Dead Zones, and Coronal Mass Ejections. ApJ530:1036-1048

Khodachenko ML, Ribas I, Lammer H et al. (2007) Coronal Mass Ejection (CME) Activity of Low Mass M Stars as An Important Factor for The Habitability of Terrestrial Exoplanets. I. CME Impact on Expected Magnetospheres of Earth-Like Exoplanets in Close-In Habitable Zones. Astrobiology 7:167-184

Khodachenko ML, Alexeev I, Belenkaya E et al. (2012) Magnetospheres of "Hot Jupiters": The Importance of Magnetodisks in Shaping a Magnetospheric Obstacle. ApJ744:70

Kislyakova KG, Holmström M, Lammer H, Odert P Khodachenko ML (2014) Magnetic moment and plasma environment of HD 209458b as determined from Ly $\alpha$ observations. Science 346:981-984

Kraft RP (1967) Studies of Stellar Rotation. V. The Dependence of Rotation on Age among SolarType Stars. ApJ150:551-+

Lai D, Helling C van den Heuvel EPJ (2010) Mass Transfer, Transiting Stream, and Magnetopause in Close-in Exoplanetary Systems with Applications to WASP-12. ApJ721:923-928

Lammer H, Selsis F, Ribas I et al. (2003) Atmospheric Loss of Exoplanets Resulting from Stellar X-Ray and Extreme-Ultraviolet Heating. ApJ598:L121-L124

Lammer H, Lichtenegger HIM, Kulikov YN et al. (2007) Coronal Mass Ejection (CME) Activity of Low Mass M Stars as An Important Factor for The Habitability of Terrestrial Exoplanets. II. CME-Induced Ion Pick Up of Earth-like Exoplanets in Close-In Habitable Zones. Astrobiology 7:185-207

Lang P, Jardine M, Morin J et al. (2014) Modelling the hidden magnetic field of low-mass stars. MNRAS439:2122-2131

Lanza AF (2009) Stellar coronal magnetic fields and star-planet interaction. A\&A505:339-350

Li SL, Miller N, Lin DNC Fortney JJ (2010) WASP-12b as a prolate, inflated and disrupting planet from tidal dissipation. Nature463:1054-1056

Lim J White SM (1996) Limits to Mass Outflows from Late-Type Dwarf Stars. ApJ462:L91+

Lim J, White SM Slee OB (1996) The Radio Properties of the dMe Flare Star Proxima Centauri. ApJ460:976-+

Lima JJG, Priest ER Tsinganos K (2001) An analytical MHD wind model with latitudinal dependences obtained using separation of the variables. A\&A371:240-249

Llama J, Vidotto AA, Jardine M et al. (2013) Exoplanet transit variability: bow shocks and winds around HD 189733b. MNRAS436:2179-2187

Lovelace RVE, Romanova MM Barnard AW (2008) Planet migration and disc destruction due to magneto-centrifugal stellar winds. MNRAS389:1233-1239

Lüftinger T, Vidotto AA Johnstone CP (2015) Magnetic Fields and Winds of Planet Hosting Stars. In: Lammer H Khodachenko M (eds) Astrophysics and Space Science Library, Astrophysics and Space Science Library, vol 411, p 37, DOI 10.1007/978-3-319-09749-7_3

Maggio A, Sanz-Forcada J Scelsi L (2011) Photospheric and coronal abundances in solar-type stars: the peculiar case of $\tau$ Bootis. A\&A527:A144

Marsden SC, Donati JF, Semel M, Petit P Carter BD (2006) Surface differential rotation and photospheric magnetic field of the young solar-type star HD 171488 (V889 Her). MNRAS370:468476 
Marsden SC, Jardine MM, Ramírez Vélez JC et al. (2011) Magnetic fields and differential rotation on the pre-main sequence - I. The early-G star HD 141943 - brightness and magnetic topologies. MNRAS413:1922-1938

Matsakos T, Uribe A Königl A (2015) Classification of magnetized star-planet interactions: bow shocks, tails, and inspiraling flows. A\&A578:A6

Matsumoto T Suzuki TK (2012) Connecting the Sun and the Solar Wind: The First 2.5-dimensional Self-consistent MHD Simulation under the Alfvén Wave Scenario. ApJ749:8

Matsumoto T Suzuki TK (2014) Connecting the Sun and the solar wind: the self-consistent transition of heating mechanisms. MNRAS440:971-986

Matt S Pudritz RE (2005) Accretion-powered Stellar Winds as a Solution to the Stellar Angular Momentum Problem. ApJ632:L135-L138

Matt SP, MacGregor KB, Pinsonneault MH Greene TP (2012) Magnetic Braking Formulation for Sun-like Stars: Dependence on Dipole Field Strength and Rotation Rate. ApJ754:L26

Mestel L (1968) Magnetic braking by a stellar wind-I. MNRAS138:359-+

Morgenthaler A, Petit P, Morin J et al. (2011) Direct observation of magnetic cycles in Sun-like stars. Astronomische Nachrichten 332:866

Morgenthaler A, Petit P, Saar S et al. (2012) Long-term magnetic field monitoring of the Sun-like star $\xi$ Bootis A. A\&A540:A138

Morin J, Donati J, Forveille T et al. (2008a) The stable magnetic field of the fully convective star V374 Peg. MNRAS384:77-86

Morin J, Donati J, Petit P et al. (2008b) Large-scale magnetic topologies of mid M dwarfs. MNRAS390:567-581

Morin J, Donati J, Petit P et al. (2010) Large-scale magnetic topologies of late M dwarfs. MNRAS407:2269-2286

Morin J, Jardine M, Reiners A et al. (2013) Multiple views of magnetism in cool stars. Astronomische Nachrichten 334:48

Nicholson BA, Vidotto AA, Mengel M et al. (2016) Temporal variability of the wind from the star $\tau$ Boötis. MNRAS459:1907-1915

Pallavicini R, Golub L, Rosner R et al. (1981) Relations among stellar X-ray emission observed from Einstein, stellar rotation and bolometric luminosity. ApJ248:279-290

Parsons SG, Marsh TR, Gänsicke BT et al. (2012) A precision study of two eclipsing white dwarf plus M dwarf binaries. MNRAS420:3281-3297

Petit P, Dintrans B, Solanki SK et al. (2008) Toroidal versus poloidal magnetic fields in Sun-like stars: a rotation threshold. MNRAS388:80-88

Petit P, Dintrans B, Morgenthaler A et al. (2009) A polarity reversal in the large-scale magnetic field of the rapidly rotating sun HD 190771. A\&A508:L9-L12

Pevtsov AA, Fisher GH, Acton LW et al. (2003) The Relationship Between X-Ray Radiance and Magnetic Flux. ApJ598:1387-1391

Pillitteri I, Wolk SJ, Lopez-Santiago J et al. (2014) The Corona of HD 189733 and its X-Ray Activity. ApJ785:145

Pinto RF, Brun AS, Jouve L Grappin R (2011) Coupling the Solar Dynamo and the Corona: Wind Properties, Mass, and Momentum Losses during an Activity Cycle. ApJ737:72-+

Pizzolato N, Maggio A, Micela G, Sciortino S Ventura P (2003) The stellar activity-rotation relationship revisited: Dependence of saturated and non-saturated X-ray emission regimes on stellar mass for late-type dwarfs. A\&A397:147-157

Pneuman GW Kopp RA (1971) Gas-Magnetic Field Interactions in the Solar Corona. Sol Phys 18:258-270

Reiners A Basri G (2008) The moderate magnetic field of the flare star Proxima Centauri. A\&A489:L45-L48

Reiners A, Basri G Browning M (2009) Evidence for Magnetic Flux Saturation in Rapidly Rotating M Stars. ApJ692:538-545

Reiners A, Schüssler M Passegger VM (2014) Generalized Investigation of the Rotation-Activity Relation: Favoring Rotation Period instead of Rossby Number. ApJ794:144 
Réville V, Brun AS, Matt SP, Strugarek A Pinto RF (2015) The Effect of Magnetic Topology on Thermally Driven Wind: Toward a General Formulation of the Braking Law. ApJ798:116

Ribas I, Guinan EF, Guedel M Audard M (2005) Evolution of the Solar Activity over Time and Effects on Planetary Atmospheres. I. High-Energy Irradiances (1-1700 Å). ApJ622:680-694

Ribas I, Porto de Mello GF, Ferreira LD et al. (2010) Evolution of the Solar Activity Over Time and Effects on Planetary Atmospheres. II. $\kappa^{1}$ Ceti, an Analog of the Sun when Life Arose on Earth. ApJ714:384

Ribas I, Bolmont E, Selsis F et al. (2016) The habitability of Proxima Centauri b. I. Irradiation, rotation and volatile inventory from formation to the present. A\&A596:A111

Saar SH (1996) Recent magnetic fields measurements of stellar. In: Strassmeier KG Linsky JL (eds) Stellar Surface Structure, IAU Symposium, vol 176, p 237

Saar SH (2001) Recent Measurements of (and Inferences About) Magnetic Fields on K and M Stars (CD-ROM Directory: contribs/saar1). In: Garcia Lopez RJ, Rebolo R Zapaterio Osorio MR (eds) 11th Cambridge Workshop on Cool Stars, Stellar Systems and the Sun, Astronomical Society of the Pacific Conference Series, vol 223, p 292

Sanz-Forcada J, Micela G, Ribas I et al. (2011) Estimation of the XUV radiation onto close planets and their evaporation. A\&A532:A6

Scandariato G, Maggio A, Lanza AF et al. (2013) A coordinated optical and X-ray spectroscopic campaign on HD 179949: searching for planet-induced chromospheric and coronal activity. A\&A552:A7

See V, Jardine M, Vidotto AA et al. (2014) The effects of stellar winds on the magnetospheres and potential habitability of exoplanets. A\&A570:A99

Shkolnik E, Bohlender DA, Walker GAH Collier Cameron A (2008) The On/Off Nature of StarPlanet Interactions. ApJ676:628-638

Skumanich A (1972) Time Scales for CA II Emission Decay, Rotational Braking, and Lithium Depletion. ApJ171:565-+

Sokolov IV, van der Holst B, Oran R et al. (2013) Magnetohydrodynamic Waves and Coronal Heating: Unifying Empirical and MHD Turbulence Models. ApJ764:23

Solanki SK (1994) Must the Magnetic Field Saturate on Rapidly Rotating Stars? In: Caillault JP (ed) Cool Stars, Stellar Systems, and the Sun, Astronomical Society of the Pacific Conference Series, vol 64, p 477

Sterenborg MG, Cohen O, Drake JJ Gombosi TI (2011) Modeling the young Sun's solar wind and its interaction with Earth's paleomagnetosphere. Journal of Geophysical Research (Space Physics) 116:A01217

Strugarek A, Brun AS, Matt SP Réville V (2015) Magnetic Games between a Planet and Its Host Star: The Key Role of Topology. ApJ815:111

Suzuki TK, Imada S, Kataoka R et al. (2013) Saturation of Stellar Winds from Young Suns. PASJ65:98

Telleschi A, Guedel M, Briggs K et al. (2005) Coronal Evolution of the Sun in Time: HighResolution X-Ray Spectroscopy of Solar Analogs with Different Ages. ApJ622:653-679

Testa P, Saar SH Drake JJ (2015) Stellar activity and coronal heating: an overview of recent results. Philosophical Transactions of the Royal Society of London Series A 373:20140,259-20140,259

Tsinganos K Low BC (1989) Steady hydromagnetic flows in open magnetic fields. II - Global flows with static zones. ApJ342:1028-1048

van den Oord GHJ Doyle JG (1997) Constraints on mass loss from dMe stars: theory and observations. A\&A319:578-588

van der Holst B, Manchester WB, Frazin RA et al. (2010) A Data-driven, Two-temperature Solar Wind Model with Alfvén Waves. ApJ725:1373-1383

van der Holst B, Sokolov IV, Meng X et al. (2014) Alfvén Wave Solar Model (AWSoM): Coronal Heating. ApJ782:81

Vidotto AA (2016) The magnetic field vector of the Sun-as-a-star. MNRAS459:1533-1542

Vidotto AA Donati JF (2017) Predicting radio emission from the newborn hot Jupiter V830 Tau and its host star. ArXiv e-prints 
Vidotto AA Jatenco-Pereira V (2006) The Effects of Alfvén Waves and Radiation Pressure in Dusty Winds of Late-Type Stars. II. Dust-Cyclotron Damping. ApJ639:416-422

Vidotto AA, Opher M, Jatenco-Pereira V Gombosi TI (2009a) Simulations of Winds of WeakLined T Tauri Stars: The Magnetic Field Geometry and the Influence of the Wind on Giant Planet Migration. ApJ703:1734-1742

Vidotto AA, Opher M, Jatenco-Pereira V Gombosi TI (2009b) Three-dimensional Numerical Simulations of Magnetized Winds of Solar-like Stars. ApJ699:441-452

Vidotto AA, Jardine M Helling C (2010a) Early UV Ingress in WASP-12b: Measuring Planetary Magnetic Fields. ApJ722:L168-L172

Vidotto AA, Opher M, Jatenco-Pereira V Gombosi TI (2010b) Simulations of Winds of Weaklined T Tauri Stars. II. The Effects of a Tilted Magnetosphere and Planetary Interactions. ApJ720:1262-1280

Vidotto AA, Jardine M Helling C (2011a) Prospects for detection of exoplanet magnetic fields through bow-shock observations during transits. MNRAS411:L46-L50

Vidotto AA, Jardine M, Opher M, Donati JF Gombosi TI (2011b) Powerful winds from low-mass stars: V374 Peg. MNRAS412:351-362

Vidotto AA, Llama J, Jardine M, Helling C Wood K (2011c) Shock formation around planets orbiting M-dwarf stars. Astronomische Nachrichten 332:1055

Vidotto AA, Fares R, Jardine M et al. (2012) The stellar wind cycles and planetary radio emission of the $\tau$ Boo system. MNRAS423:3285-3298

Vidotto AA, Jardine M, Morin J et al. (2013) Effects of M dwarf magnetic fields on potentially habitable planets. A\&A557:A67

Vidotto AA, Gregory SG, Jardine M et al. (2014a) Stellar magnetism: empirical trends with age and rotation. MNRAS441:2361-2374

Vidotto AA, Jardine M, Morin J et al. (2014b) M-dwarf stellar winds: the effects of realistic magnetic geometry on rotational evolution and planets. MNRAS438:1162-1175

Vidotto AA, Fares R, Jardine M, Moutou C Donati JF (2015) On the environment surrounding close-in exoplanets. MNRAS449:4117-4130

Vidotto AA, Donati JF, Jardine M et al. (2016) Could a change in magnetic field geometry cause the break in the wind-activity relation? MNRAS455:L52-L56

Villadsen J, Hallinan G, Bourke S, Guedel M Rupen M (2014) First Detection of Thermal Radio Emission from Solar-type Stars with the Karl G. Jansky Very Large Array. ApJ788:112

Waite IA, Marsden SC, Carter BD et al. (2011) Magnetic fields and differential rotation on the pre-main sequence - III. The early-G star HD 106506. MNRAS413:1949-1960

Waite IA, Marsden SC, Carter BD et al. (2015) Magnetic fields on young, moderately rotating Sun-like stars - I. HD 35296 and HD 29615. MNRAS449:8-24

Waite IA, Marsden SC, Carter BD et al. (2017) Magnetic fields on young, moderately rotating Sun-like stars - II. EK Draconis (HD 129333). MNRAS465:2076-2091

Wargelin BJ Drake JJ (2002) Stringent X-Ray Constraints on Mass Loss from Proxima Centauri. ApJ578:503-514

Washimi H Shibata S (1993) Thermo-centrifugal wind from a rotating magnetic dipole. MNRAS262:936-944

West AA, Hawley SL, Bochanski JJ et al. (2008) Constraining the Age-Activity Relation for Cool Stars: The Sloan Digital Sky Survey Data Release 5 Low-Mass Star Spectroscopic Sample. AJ135:785-795

Wood BE (2004) Astrospheres and Solar-like Stellar Winds. Living Reviews in Solar Physics 1:2$+$

Wood BE Linsky JL (2010) Resolving the $\xi$ Boo Binary with Chandra, and Revealing the Spectral Type Dependence of the Coronal "FIP Effect". ApJ717:1279-1290

Wood BE, Linsky JL, Müller H Zank GP (2001) Observational Estimates for the Mass-Loss Rates of $\alpha$ Centauri and Proxima Centauri Using Hubble Space Telescope Ly $\alpha$ Spectra. ApJ547:L49-L52

Wood BE, Müller HR, Zank GP Linsky JL (2002) Measured Mass-Loss Rates of Solar-like Stars as a Function of Age and Activity. ApJ574:412-425 
Wood BE, Müller HR, Zank GP, Linsky JL Redfield S (2005) New Mass-Loss Measurements from Astrospheric Ly $\alpha$ Absorption. ApJ628:L143-L146

Wood BE, Müller HR, Redfield S Edelman E (2014) Evidence for a Weak Wind from the Young Sun. ApJ781:L33

Wright NJ, Drake JJ, Mamajek EE Henry GW (2011) The Stellar-activity-Rotation Relationship and the Evolution of Stellar Dynamos. ApJ743:48

Yang SH, Zhang J, Jin CL, Li LP Duan HY (2009) Response of the solar atmosphere to magnetic field evolution in a coronal hole region. A\&A501:745-753

Zendejas J, Segura A Raga AC (2010) Atmospheric mass loss by stellar wind from planets around main sequence $M$ stars. Icarus210:539-544

Zieger B, Vogt J Glassmeier KH (2006) Scaling relations in the paleomagnetosphere derived from MHD simulations. Journal of Geophysical Research (Space Physics) 111:A06203

Zuluaga JI, Bustamante S, Cuartas PA Hoyos JH (2013) The Influence of Thermal Evolution in the Magnetic Protection of Terrestrial Planets. ApJ770:23 